PROCEEDINGS OF THE

AMERICAN MATHEMATICAL SOCIETY

Volume 136, Number 10, October 2008, Pages 3449-3459

S 0002-9939(08)09395-7

Article electronically published on May 16, 2008

\title{
GALOIS COHOMOLOGY OF COMPLETED LINK GROUPS
}

\author{
INGA BLOMER, PETER A. LINNELL, AND THOMAS SCHICK
}

(Communicated by Martin Lorenz)

\begin{abstract}
In this paper we compute the Galois cohomology of the pro- $p$ completion of primitive link groups. Here, a primitive link group is the fundamental group of a tame link in $S^{3}$ whose linking number diagram is irreducible modulo $p$ (e.g. none of the linking numbers is divisible by $p$ ).

The result is that (with $\mathbb{Z} / p \mathbb{Z}$-coefficients) the Galois cohomology is naturally isomorphic to the $\mathbb{Z} / p \mathbb{Z}$-cohomology of the discrete link group.

The main application of this result is that for such groups the Baum-Connes conjecture or the Atiyah conjecture are true for every finite extension (or even every elementary amenable extension), if they are true for the group itself.
\end{abstract}

\section{INTRODUCTION}

Throughout the paper we fix a prime number $p$. If $G$ is a discrete group, then $\hat{G}$ will denote its pro- $p$ completion.

1.1. Definition. We denote the fundamental group $G$ of the complement $M$ of a tubular neighborhood of a tame link $L$ with $d$ components in $S^{3}$ as a link group with $d$ components. We define the linking diagram to be the edge-labeled graph whose vertices are the components of the link. Any two vertices are joined by one edge, which is labeled with the linking number of the two link components involved.

We say the link group $G$ is $q$-primitive for a prime $q$ if there is a spanning subtree of the linking diagram such that none of the labels of the edges of this subtree is congruent to 0 modulo $p$. It is called primitive if it is $q$-primitive for every prime $q$.

Observe that in particular every knot group (i.e. a link group of a link with only one component) is primitive, because there are no edges to worry about.

Labute computes in 6 , the lower central series quotients of primitive link groups. He shows in particular that all of them are torsion free. He uses Lie algebra techniques developed in [9, 10, 11. These techniques give results not only about the lower central series quotients, but also about the pro- $p$ completions and their Galois cohomology.

In [13, 17] it became important to compute the Galois cohomology of such groups because of the following result which combines special cases of 13 , Theorem 4.60, Corollary 4.62] or of [17, Theorem 10].

Received by the editors September 4, 2007.

2000 Mathematics Subject Classification. Primary 20E18; Secondary 20J06, 57M25.

Key words and phrases. Link group, Lie algebra, Galois cohomology.

The third author was funded by the DAAD (German Academic Exchange Agency).

(C)2008 American Mathematical Society Reverts to public domain 28 years from publication 
1.2. Theorem. Let $G$ be a discrete group with finite classifying space. Assume that all the lower central series quotients of $G$ are torsion free and that for every prime $q$ the canonical map

$$
H^{*}(\hat{G}, \mathbb{Z} / q) \rightarrow H^{*}(G, \mathbb{Z} / q)
$$

between the Galois cohomology of the pro-q completion $\hat{G}$ and the ordinary cohomology of $G$ is an isomorphism.

If the Atiyah conjecture holds for $G$, then it also holds for every elementary amenable extension of $G$, and in particular every finite extension of $G$.

If the Baum-Connes conjecture (with coefficients) holds for $G$, then it also holds for every elementary amenable extension of $G$, and in particular every finite extension of $G$.

Here, the Atiyah conjecture for a torsion-free group $G$ asserts that the $L^{2}$-Betti numbers of every compact manifold with fundamental group $G$ are integers. This implies that the ring $\mathbb{Q} G$ embeds into a skew field (there are versions which imply the same for $\mathbb{C} G$ ).

It is checked in the proof of [13, Proposition 5.34] that a primitive link group fulfills all the assumptions of Theorem [1.2, except for the computation of the Galois cohomology. This will be done in the present paper. Our main result is the following.

1.4. Theorem. Let $G$ be a primitive link group. Then the map

$$
H^{n}(\hat{G}, \mathbb{Z} / p) \rightarrow H^{n}(G, \mathbb{Z} / p)
$$

is an isomorphism for every $n \in \mathbb{Z}$ and every prime number $p$.

1.5. Definition. As in [13, Definition 4.3], we refer to the above property, where $H^{n}(\hat{G}, \mathbb{Z} / p) \rightarrow H^{n}(G, \mathbb{Z} / p)$ is an isomorphism for every $n \in \mathbb{Z}$ and every prime number $p$, as $G$ being cohomologically complete.

1.6. Corollary. Let $G$ be a primitive link group. If $G$ satisfies the Atiyah conjecture, then so does every elementary amenable extension of $G$, and in particular every finite extension of $G$.

The Baum-Connes conjecture is true for every elementary amenable extension of $G$, and in particular every finite extension of $G$.

Proof. All these statements follow immediately from Theorems 1.4 and 1.2, and from the fact that the Baum-Connes conjecture with coefficients is known for fundamental groups of Haken 3-manifolds; compare e.g. [16, Theorem 5.23] or [14, Theorem 5.2].

This work was inspired by the confirmation of John Labute that the homomorphism of (1.3) indeed should be an isomorphism for primitive link groups, and it is very much based on Labute's work cited above. Since the result does not seem to be available in the literature, and since it is not obvious how to prove it using 9, 10, 11, 6], we indicate the proof in this paper. We will heavily refer to results and methods which can be found in these papers. Alternatively, one could have used a suitable version of the mildness and appropriate versions of the results of 7 . However, since there the $p$-central series is in the focus whereas we use the central series, we try to give a somewhat independent treatment. 


\section{Proof of Theorem 1.4}

First we observe (cf. [13, Lemma 4.5]):

2.1. Lemma. Let $G$ be a discrete group. Then

$$
H^{k}(\hat{G}, \mathbb{Z} / p) \rightarrow H^{k}(G, \mathbb{Z} / p)
$$

is an isomorphism for $k=0$ and $k=1$.

Proof. For $k=0$ this is immediately clear from the definition. Also, $H^{1}(G, \mathbb{Z} / p)=$ $\operatorname{Hom}(G, \mathbb{Z} / p)$ and $H^{1}(\hat{G}, \mathbb{Z} / p)=\operatorname{Hom}(\hat{G}, \mathbb{Z} / p)$. But by the very definition of the pro- $p$ completion every homomorphism from $G$ to $\mathbb{Z} / p$ extends uniquely to a homomorphism $\hat{G} \rightarrow \mathbb{Z} / p$.

2.2. Example. Let $F$ be an arbitrary free group (not necessarily finitely generated). Then $F$ is cohomologically complete, as defined in Definition 1.5 .

Proof. Since $F$ has cohomological dimension $\leq 1$, in view of Lemma 2.1 it suffices to prove that the pro- $p$ completion of $F$ has cohomological dimension $\leq 1$, i.e. that $\hat{F}^{p}$ is a free pro- $p$ group. But this follows from [19, Corollary 4 and Remark on p. 8].

The first step in proving Theorem 1.4 is the computation of the cohomology of the discrete link group:

2.3. Lemma. Assume that $G$ is a primitive link group with $d$ components. Then $G$ has a compact 2-dimensional classifying space and

$$
\operatorname{dim}_{\mathbb{Z} / p \mathbb{Z}} H^{n}(G, \mathbb{Z} / p \mathbb{Z})= \begin{cases}1, & \text { if } n=0, \\ d, & \text { if } n=1, \\ d-1, & \text { if } n=2, \\ 0, & \text { if } n>2 .\end{cases}
$$

Proof. Let $L$ be a link with $d$ components with a primitive linking diagram such that $G$ is the fundamental group of $S^{3}-L$. It is shown in the proof of [13, Proposition 5.34 ] that the compact 3 -dimensional manifold with boundary $S^{3}-U(L)(U(L)$ being an open tubular neighborhood of $L$ ) is a classifying space for $G$, homotopy equivalent to a 2-dimensional CW-complex. In particular,

$$
H^{n}(G, \mathbb{Z} / p \mathbb{Z})=H^{n}\left(S^{3}-L, \mathbb{Z} / p \mathbb{Z}\right),
$$

which can easily be computed using Alexander duality and gives the asserted dimensions.

These computations show that the following proposition is relevant for $p$-primitive link groups.

2.4. Proposition. Let $G$ be a discrete group with finite classifying space of dimension 2. Then

$$
H^{n}(\hat{G}, \mathbb{Z} / p) \rightarrow H^{n}(G, \mathbb{Z} / p) \text { is an isomorphism for every } n \in \mathbb{Z}
$$

if and only if

(1) $H^{3}(\hat{G}, \mathbb{Z} / p \mathbb{Z})=0$,

(2) $\operatorname{dim}_{\mathbb{Z} / p \mathbb{Z}} H^{2}(\hat{G}, \mathbb{Z} / p \mathbb{Z})=\operatorname{dim}_{\mathbb{Z} / p \mathbb{Z}} H^{2}(G, \mathbb{Z} / p \mathbb{Z})$. 
Condition (11) is equivalent to the fact that the cohomological dimension of $\hat{G}$ is $\leq 2$. If $\hat{G}=F / R$, where $F$ is a finitely generated free pro-p group, then this is also equivalent to $R / \overline{[R, R]}$ (where $\overline{[R, R]}$ is the closure of the subgroup generated by all commutators of $R$ ) being a free $\mathbb{Z}_{p}[[\hat{G}]]$-module. $\mathbb{Z}_{p}[[\hat{G}]]$ is the completed group ring (compare [2]), and the action is induced by conjugation.

Proof. (2.5) obviously implies that (11) and (2) hold.

By [19. Propositions 11 and 20] we have $H^{3}(\hat{G}, \mathbb{Z} / p)=0$ if and only if the cohomological dimension of $\hat{G}$ is $\leq 2$. The equivalence of the last condition with (11) follows immediately from [2, Theorem 5.2 and Corollary 5.3]. Here we use the fact that the abelian group $N_{p}:=R / \overline{[R, R]}$ is equal to its $p$-Sylow subgroup since a free pro- $p$ group (like $R$ ) does not have non-trivial cyclic quotients of prime order except for $\mathbb{Z} / p$, so that our definition indeed coincides with the definition of [2].

We use [2, Lemma 4.2] to compute the Galois cohomology of $\hat{G}$ using a $\mathbb{Z}_{p}[[\hat{G}]]$ resolution of the $p$-adic integers $\mathbb{Z}_{p}$.

We change notation and write $G=F / R$ with a free group $F$ on $d$ generators $x_{1}, \ldots, x_{d}$ and relations $R$ (normally generated by $r$ relators $r_{1}, \ldots, r_{r}$, and where $r$ is minimal with this property), where the presentation is given by a finite classifying 2-complex $X$ for $G$.

Then $\hat{G}=\hat{F} / \bar{R}$, where $\bar{R}$ is the closed normal subgroup of $\hat{F}$ generated by $R$ (compare [13, Lemma 3.9]).

In the proof of [2, Theorem 5.2] the exact sequence

$$
0 \rightarrow N_{p} \rightarrow I(F) \hat{\otimes}_{\mathbb{Z}_{p}\left[\left[N_{p}\right]\right]} \mathbb{Z}_{p} \rightarrow \mathbb{Z}_{p}[[\hat{G}]] \rightarrow \mathbb{Z}_{p} \rightarrow 0
$$

is given. Since $F$ has cohomological dimension 1, the discussion at the beginning of [2, Proof of Corollary 5.3] shows that $I(F)$ is a free $\mathbb{Z}_{p}[[\hat{F}]]$-module generated by $\left\{\left(1-x_{1}\right), \ldots,\left(1-x_{d}\right)\right\}$ (note that $\left\{x_{1}, \ldots, x_{d}\right\}$ also constitutes a set of free generators for $\hat{F})$. This fact is also used in [9, Example (2) on p. 144]. Hence $I(F) \hat{\otimes}_{\mathbb{Z}_{p}\left[\left[N_{p}\right]\right]} \mathbb{Z}_{p} \cong \mathbb{Z}_{p}[[\hat{G}]]^{d}$.

As mentioned by Brumer in [2, his reasoning also applies to the discrete group $G$ to give the exact sequence

$$
0 \rightarrow N \stackrel{d_{2}}{\longrightarrow} \mathbb{Z}[G]^{d} \stackrel{d_{1}}{\longrightarrow} \mathbb{Z}[G] \stackrel{d_{0}}{\longrightarrow} \mathbb{Z} \rightarrow 0,
$$

where $N=R /[R, R]$ is the abelianization of the relations. It follows from the construction that the inclusions $G \rightarrow \hat{G}$ and $\mathbb{Z} \hookrightarrow \mathbb{Z}_{p}$ induce maps which are just the inclusion of a free $\mathbb{Z}[G]$-module into its completion for the two middle terms in the exact sequences. Moreover, the boundary map $d_{1}: \mathbb{Z}[G]^{d} \rightarrow \mathbb{Z}[G]$ maps the generator of copy number $j$ of $\mathbb{Z}[G]$ to $1-x_{j}$, where $x_{j}$ is (the image of) one of the generators of $F$.

On the other hand, the (universal covering of the) 2-complex $X$ has a cellular chain complex

$$
0 \rightarrow \mathbb{Z}[G]^{r} \stackrel{d_{2}^{X}}{\longrightarrow} \mathbb{Z}[G]^{d} \stackrel{d_{1}^{X}}{\longrightarrow} \mathbb{Z}[G] \stackrel{d_{0}^{X}}{\longrightarrow} \mathbb{Z} \rightarrow 0 .
$$

Since this universal covering is contractible by assumption, this sequence is exact (this is the reason why we added the group $\mathbb{Z}$ at the end). Moreover, the differentials $d_{1}^{X}$ and $d_{1}$ as well as $d_{0}^{X}$ and $d_{0}$ coincide. Identifying $N$ and $\mathbb{Z}[G]^{r}$ with their images under $d_{2}$ or $d_{2}^{X}$, respectively, we see that they are isomorphic, in particular $N$ is a free $\mathbb{Z}[G]$-module, freely generated by the relators $r_{1}, \ldots, r_{r}$. To compare the sequence (2.7) with (2.6) we observe that by [2, Corollary 5.3] (under the 
assumption that $\hat{G}$ has cohomological dimension $\leq 2) N_{p}$ is a free $\mathbb{Z}_{p}[[\hat{G}]]$-module, not necessarily generated by all the $r_{1}, \ldots, r_{r}$, but instead only by a minimal subset which still normally and topologically generates $\bar{R}$ (which a priori might be smaller). By [2, Proof of Corollary 5.3], we need any subset whose image in $\bar{R} / \overline{[R, F] R^{p}}$ is a basis as a $\mathbb{Z} / p \mathbb{Z}$-vector space.

To compute the cohomology with $\mathbb{Z} / p \mathbb{Z}$-coefficients of $G$ or $\hat{G}$, respectively, we have to pass to the (topological) dual chain complexes and take their cohomology.

By the very definition of the completion, $\operatorname{Hom}\left(\mathbb{Z}[G]^{d}, \mathbb{Z} / p \mathbb{Z}\right)$ is isomorphic to $\operatorname{Hom}\left(\mathbb{Z}_{p}[[\hat{G}]]^{d}, \mathbb{Z} / p \mathbb{Z}\right)$ (every such homomorphism extends uniquely). However, the same considerations only show that $\operatorname{Hom}\left(N_{p}, \mathbb{Z} / p \mathbb{Z}\right) \rightarrow \operatorname{Hom}(N, \mathbb{Z} / p \mathbb{Z})$ is injective. Diagram chasing reveals that the induced maps $H^{j}(\hat{G}, \mathbb{Z} / p \mathbb{Z}) \rightarrow H^{j}(G, \mathbb{Z} / p \mathbb{Z})$ are isomorphisms for $j=0,1$ and injective for $j=2$. Since we assume that for $j=2$ the two cohomology groups have the same finite dimension, the map is an isomorphism in this case, as well.

To apply Proposition 2.4, we next derive a presentation of the pro- $p$ completion of link groups.

2.8. Proposition. Let $G$ be a link group with $d$ components. Then the pro-p completion $\hat{G}$ of $G$ has a presentation as a pro-p group with $d$ generators and $d-1$ relators. In other words, $\hat{G}$ is a quotient of a free pro-p group $F$ with $d$ generators, and the kernel $R$ is the closure of the normal subgroup generated by $d-1$ elements.

If, moreover, $G$ is primitive and if the $\mathbb{Z}_{p}[[\hat{G}]]$-module $R / \overline{[R, R]}$ is free on $d-1$ generators, then the natural map

$$
H^{n}(\hat{G}, \mathbb{Z} / p \mathbb{Z}) \rightarrow H^{n}(G, \mathbb{Z} / p \mathbb{Z})
$$

is an isomorphism for every $n \in \mathbb{Z}$.

Proof. By a result of Milnor (compare [6, p. 951]), in our situation the lower central series quotients $G / \gamma_{n}(G)$ have presentations

$$
\left\langle x_{1}, \ldots, x_{d} \mid\left[x_{1}, w_{1}^{(n)}\right]=\cdots=\left[x_{d-1}, w_{d-1}^{(n)}\right]=1, \gamma_{n}(F)\right\rangle
$$

where $F$ is the free group generated by $x_{1}, \ldots, x_{d},[x, y]=x^{-1} y^{-1} x y$ is the commutator, and for $k=1, \ldots, d-1$ and $n \in \mathbb{Z}$ we have certain $w_{k}^{(n)} \in F$ which in addition fulfill $w_{k}^{(n)} \equiv w_{k}^{(n+1)}$ modulo $\gamma_{n}(F)$.

Consequently, if $\gamma_{n}^{p}(G)$ is the lower $p$-central series (defined inductively by $\gamma_{1}^{p}(G)$ $=G$ and $\left.\gamma_{n+1}^{p}(G)=\left(\gamma_{n}(G)\right)^{p}\left[\gamma_{n}^{p}(G), G\right]\right)$, then $w_{k}^{(n)} \equiv w_{k}^{(n+1)}$ modulo $\gamma_{n}^{p}(F)$, and $G / \gamma_{n}^{p}(G)$ has the presentation

$$
\left\langle x_{1}, \ldots, x_{d} \mid\left[x_{1}, w_{1}^{(n)}\right]=\cdots=\left[x_{d-1}, w_{d-1}^{(n)}\right]=1, \gamma_{n}^{p}(F)\right\rangle .
$$

Since $\hat{G}$ is the inverse limit of the inverse system given by $G / \gamma_{n}^{p}(G)$, it follows that for each $k=1, \ldots, d$ the sequence $w_{k}^{(n)}$ converges in $\hat{G}$ to $w_{k}$ and that a presentation of $\hat{G}$ as pro- $p$ group is given by

$$
\left\langle x_{1}, \ldots, x_{d} \mid\left[x_{1}, w_{1}\right]=\cdots=\left[x_{d-1}, w_{d-1}\right]=1\right\rangle .
$$

Assume now that $G$ is primitive. By Lemma $2.3 G$ has a classifying space of dimension 2. Because of the assumptions and Proposition 2.4 we only have to check that $\operatorname{dim}_{\mathbb{Z} / p} H^{2}(G, \mathbb{Z} / p)=\operatorname{dim}_{\mathbb{Z} / p} H^{2}(\hat{G}, \mathbb{Z} / p)$. In the proof of Proposition 2.4 we have seen that the cohomology of $\hat{G}$ is the cohomology of the following cochain 
complex (we dualize (2.6) using the fact that $I(F)$ is a free $\mathbb{Z}_{p}[[\hat{G}]]$-module on $d$ generators and by the assumption that $N_{p}=R / \overline{[R, R]}$ is free on $d-1$ generators)

$$
0 \leftarrow(\mathbb{Z} / p \mathbb{Z})^{d-1} \stackrel{d_{1}^{*}}{\longleftarrow}(\mathbb{Z} / p \mathbb{Z})^{d} \stackrel{d_{0}^{*}}{\longleftarrow} \mathbb{Z} / p \mathbb{Z} .
$$

By Lemma 2.1 and Lemma 2.3 we know that $\operatorname{dim}_{\mathbb{Z} / p \mathbb{Z}} H^{0}(\hat{G}, \mathbb{Z} / p \mathbb{Z})=1$ and $\operatorname{dim}_{\mathbb{Z} / p \mathbb{Z}} H^{1}(\hat{G}, \mathbb{Z} / p \mathbb{Z})=d$. This implies that $d_{1}^{*}$ in the cochain complex (2.9) is trivial, and therefore $\operatorname{dim}_{\mathbb{Z} / p \mathbb{Z}} H^{2}(\hat{G}, \mathbb{Z} / p \mathbb{Z})=d-1=\operatorname{dim}_{\mathbb{Z} / p \mathbb{Z}} H^{2}(G, \mathbb{Z} / p \mathbb{Z})$.

Note that it is crucial not only to know that $R / \overline{[R, R]}$ is free, but that it is free on $d-1$ free generators.

2.10. Remark. It should be observed that it is not true in general that a discrete link group has a presentation with $d$ generators and $d-1$ relators, so that the presentation obtained in Proposition 2.8 will in general not come from a presentation of $G$ itself.

In order to show that primitive link groups are cohomologically complete, it remains to show that for a primitive $d$-component link group $G$ with pro- $p$ completion $\hat{G}=F / R$ the following holds:

$$
R / \overline{[R, R]} \text { is a free } \mathbb{Z}_{p}[[\hat{G}]] \text {-module on }(d-1) \text {-generators. }
$$

From now on let $F$ be a finitely generated free pro- $p$ group and $R$ a closed subgroup of $F$. Set $G:=F / R$. We now give a criterion in terms of filtrations, graded rings and graded modules for $N:=R / \overline{[R, R]}$ to be a free $\mathbb{Z}_{p}[[G]]$-module.

Observe that the lower central series $\gamma_{1}(F)=F, \gamma_{k}(F)=\overline{\left[\gamma_{k-1}(F), F\right]}$ provides a filtration of $F$ by closed subgroups, which induces a filtration $N_{k}$ of $N$, where $N_{k}$ is the image of $R \cap \gamma_{k}(F)$ under the projection $R \rightarrow N$.

This induces graded groups $\operatorname{gr}(F)$ with $\operatorname{gr}(F)_{k}:=\gamma_{k}(F) / \gamma_{k+1}(F)$. Each $\operatorname{gr}(F)_{k}$ is an abelian pro- $p$ group, i.e. a $\mathbb{Z}_{p}$-module. Moreover, the commutator induces a Lie ring structure on the direct $\operatorname{sum} \operatorname{gr}(F)$ of all the $\operatorname{gr}(F)_{k}$, so that $\operatorname{gr}(F)$ is a $\mathbb{Z}_{p^{-L i e}}$ algebra (compare [8, Section 2.1], ). By [8, Proposition 1] $\operatorname{gr}(F)$ is a free Lie algebra over $\mathbb{Z}_{p}$ generated by the image of the free (topological) generators of $F$. Similarly, we define $\operatorname{gr}(N)$ by $\operatorname{gr}(N)_{k}:=N_{k} / N_{k+1}$. Observe that $\operatorname{gr}(N)$ inherits a $\mathbb{Z}_{p}$-Lie algebra structure, but it is an abelian Lie algebra (i.e. the bracket is trivial).

Let $I$ be the augmentation ideal of $\mathbb{Z}_{p}[[G]]$, i.e. the kernel of the augmentation homomorphism $\mathbb{Z}_{p}[[G]] \rightarrow \mathbb{Z}_{p}$. Then the closures of the powers of $I$ provide a filtration of $\mathbb{Z}_{p}[[G]]$ by closed ideals, and we get the graded ring $\operatorname{gr}\left(\mathbb{Z}_{p}[[G]]\right)$ with $\operatorname{gr}\left(\mathbb{Z}_{p}[[G]]\right)_{k}=\overline{I^{k}} / \overline{I^{k+1}}(k=0,1, \ldots)$. It is easy to check that the $\mathbb{Z}_{p}[[G]]$-module structure of $N$ (induced from conjugation of $G$ on $R$ ) fulfills $\overline{I^{n}} N_{k} \subset N_{n+k}$. Therefore, it induces a $\operatorname{gr}\left(\mathbb{Z}_{p}[[G]]\right)$-module structure on $\operatorname{gr}(N)$.

2.11. Lemma. Assume, in the above situation, that $w_{1}, \ldots, w_{r}$ are elements of $N$ with images $W_{1}, \ldots, W_{r}$ in $\operatorname{gr}(N)$. Assume further that $\operatorname{gr}(N)$ is a free $\operatorname{gr}\left(\mathbb{Z}_{p}[[G]]\right)$ module with basis $W_{1}, \ldots, W_{r}$. Then $N$ is a free $\mathbb{Z}_{p}[[G]]$-module with basis $w_{1}, \ldots$, $w_{r}$.

Proof. The elements $w_{1}, \ldots, w_{r}$ determine a $\mathbb{Z}_{p}[[G]]$-module map $\phi: \mathbb{Z}_{p}[[G]]^{r} \rightarrow N$. We want to show that $\phi$ is an isomorphism.

An easy induction and the assumptions imply that the induced map

$$
\phi_{k}:\left(\mathbb{Z}_{p}[[G]] / \overline{I^{k-1}}\right)^{r} \rightarrow N / N_{k}
$$


is an isomorphism for every $k \geq 1$. It follows from [4, Lemme 1] that $\phi$ induces an isomorphism

$$
\phi_{\infty}:\left(\mathbb{Z}_{p}[[G]] / I^{\infty}\right)^{r} \stackrel{\cong}{\rightrightarrows} N / N_{\infty}
$$

where $I^{\infty}=\bigcap_{k \in \mathbb{N}} \overline{I^{k}}$ and $N_{\infty}=\bigcap_{k \in \mathbb{N}} N_{k}$ (here $\mathbb{N}$ denotes the positive integers $\{1,2, \ldots\})$.

Let $J$ be the radical of $\mathbb{Z}_{p}[[G]]$ (i.e. the kernel of the projection $\mathbb{Z}_{p}[[G]] \rightarrow \mathbb{Z} / p \mathbb{Z}$ ). Then certainly $I \subset J$. By [12, Chap. II (2.2.2)] $J^{\infty}$ is trivial; therefore the same is true for $I^{\infty}$.

It is well known that $\bigcap_{k \in \mathbb{N}} \gamma_{k}(F)=\{1\}$ and therefore $\bigcap_{k \in \mathbb{N}}\left(R \cap \gamma_{k}(F)\right)=\{1\}$. Assume that $w \in N_{\infty}$. Since $R \cap \gamma_{k}(F)$ maps onto $N_{k}$, for each $k \geq 1$ we find $r_{k} \in R \cap \gamma_{k}(F)$ such that the image of $r_{k}$ in $N_{k}$ is equal to $w$. Compactness and completeness of $F$ (and $R$ ) imply that we find a subsequence of $r_{k}$ which converges to $r \in R$. Since $r_{j} \in \gamma_{k}(F)$ for $j \geq k$ and since $\gamma_{k}(F)$ is closed, $r \in \gamma_{k}(F)$ for every $k$. It follows that $r$ is trivial. Because of continuity of the projection map $R \rightarrow N$, the image of $r$ in $N$ is $w$. Hence $w$ and therefore $N_{\infty}$ is trivial.

Therefore (2.12) proves the lemma.

The definition of the lower central series of a group and the corresponding graded ring also makes sense in the discrete case, and similarly with all the other objects defined above. We get a parallel theory, with the completed group ring replaced by the group ring over $\mathbb{Z}$. The discrete situation was studied thoroughly by Labute. We now give a connection between the discrete and the pro- $p$ completed versions. In particular, this will give a condition when $\operatorname{gr}(N)$ is a free $\operatorname{gr}\left(\mathbb{Z}_{p}[[G]]\right)$-module.

To do this, we recall the following lemma, whose proof we give for the convenience of the reader.

2.13. Lemma. Let $\Gamma$ be a discrete group and $i: \Gamma \rightarrow G$ be an injective group homomorphism of $\Gamma$ into a pro-p group $G$ with dense image. Then $G$ is the pro-p completion of $\Gamma$.

Proof. By the universal property of the pro- $p$ completion $\hat{\Gamma}$ we have a factorization of $i$ as $\Gamma \rightarrow \hat{\Gamma} \stackrel{I}{\rightarrow} G$. Here $I$ is surjective, since the image is closed and dense. We identify $\Gamma$ with its image in $\hat{\Gamma}$ and in $G$. It remains to check injectivity of $I$. Note that every homomorphism of $\Gamma$ onto a finite $p$-group extends uniquely to a homomorphism of $\hat{\Gamma}$. It follows in particular that for a closed normal subgroup $U$ of $\hat{\Gamma}$ of finite index $p^{n}$ the intersection with $\Gamma$ has index $p^{n}$ in $\Gamma$. Since $I$ is surjective and $U$ is compact, $I(U)$ is a closed normal subgroup of $G$ of index not bigger than $p^{n}$. Since $i$ is injective, $I(U) \cap \Gamma$ has index $p^{n}$ in $\Gamma$. It follows that the index of $I(U)$ in $G$ is precisely $p^{n}$. Therefore the induced map $I_{U}: \hat{\Gamma} / U \rightarrow G / I(U)$ is an isomorphism. By [4, Lemme 1] $I$ induces an isomorphism of $\hat{\Gamma}$ onto a quotient of $G$. It follows that $I$ itself is an isomorphism.

Let $L$ be a free $\mathbb{Z}$-Lie algebra on $d$ generators. It is a classical fact that this is the graded Lie algebra of the lower central series of a discrete free group $\Gamma_{d}$ on $d$ generators (compare [18, Theorem 6.1, LA 4.10]). If $F$ is a free pro- $p$ group on $d$ generators, then we have a canonical map $L \rightarrow \operatorname{gr}(F)$ (induced from the map $\Gamma_{d} \rightarrow F$ given by the generators), and since $\operatorname{gr}(F)$ is a free $\mathbb{Z}_{p}$-Lie algebra on the given set of generators, for each $k \in \mathbb{N} L_{k} \rightarrow \operatorname{gr}(F)_{k}$ is just the pro- $p$ completion of the (finitely generated; compare e.g. [15, Corollary 5.12]) abelian group $L_{k}$. It 
is also possible to conclude this from the fact that $\Gamma_{d} / \gamma_{k}\left(\Gamma_{d}\right)$ injects into its pro- $p$ completion by [5, Theorem 2.2] together with Lemma 2.13.

The bracket $L_{k} \times L_{r} \rightarrow L_{k+r}$ extends by continuity to $\operatorname{gr}(F)_{k} \times \operatorname{gr}(F)_{r} \rightarrow$ $\operatorname{gr}(F)_{k+r}$. This follows because the bracket is induced from taking commutators, i.e. from group multiplication, and the latter is continuous (we then only have to pass to quotients).

2.14. Lemma. Let $w_{1}, \ldots, w_{r}$ be elements of $F$ which topologically generate a closed normal subgroup $R$ of $F$. Let $W_{1}, \ldots, W_{r}$ be the natural images in $\operatorname{gr}(F)$. Assume that the set $\left\{W_{1}, \ldots, W_{r}\right\}$ belongs to the $\mathbb{Z}$-Lie subalgebra $L$ of $\operatorname{gr}(F)$. Let $\mathfrak{r}$ be the (graded) $\mathbb{Z}$-Lie ideal of $L$ generated by $\left(W_{1}, \ldots, W_{r}\right)$, and let $\mathfrak{R}$ be the closed (graded) $\mathbb{Z}_{p}$-Lie ideal of $\operatorname{gr}(F)$ generated by $\left(W_{1}, \ldots, W_{r}\right)$ (closed means each $\mathfrak{R}_{k}$ is closed in $\left.\operatorname{gr}(F)_{k}\right)$.

In this situation, $\mathfrak{R}_{k}$ is the closure of $\mathfrak{r}_{k}$ in $\operatorname{gr}(F)_{k}$. Moreover, $(\operatorname{gr}(F) / \mathfrak{R})_{k}$ is the completion of $(L / \mathfrak{r})_{k}$, where the bracket is extended by continuity. In particular, if $L / \mathfrak{r}$ is a free $\mathbb{Z}$-module, then $\operatorname{gr}(F) / \mathfrak{R}$ is a free $\mathbb{Z}_{p}$-module.

Proof. The closure of $\mathfrak{r}$ is obviously contained in $\mathfrak{R}$. Because the bracket is continuous, $\operatorname{gr}(F) \cdot \mathfrak{r}=\bar{L} \cdot \mathfrak{r} \subset \overline{L r} \subset \overline{\mathfrak{r}}$.

We have the exact sequence $0 \rightarrow \mathfrak{r}_{k} \rightarrow L_{k} \rightarrow(L / \mathfrak{r})_{k} \rightarrow 0$. E.g. by [13, Lemma $3.6]$ the pro- $p$ completion gives rise to a new exact sequence

$$
0 \rightarrow \mathfrak{R}_{k} \rightarrow \operatorname{gr}(F)_{k} \rightarrow(\operatorname{gr}(F) / \mathfrak{R})_{k} \rightarrow 0 .
$$

Here we made use of the fact that we know the completion of $L_{k}$ and the closure of $\mathfrak{r}_{k}$. The assertion of the cited lemma of course is that the quotient $(\operatorname{gr}(F) / \mathfrak{R})_{k}$ is the completion of $(L / \mathfrak{r})_{k}$. The bracket is known to be continuous; therefore it extends by continuity.

Let $U$ be the enveloping algebra of $L / \mathfrak{r}$ and $V$ be the enveloping algebra of $\operatorname{gr}(F) / \mathfrak{R}$. Then $\mathfrak{r} /[\mathfrak{r}, \mathfrak{r}]$ is a $U$-module via the adjoint action, and $\mathfrak{R} / \overline{[\mathfrak{R}, \mathfrak{R}]}$ is a $V$-module via the adjoint action (the closure again is taken degree-wise).

Assume that $L / \mathfrak{r}$ is a free $\mathbb{Z}$-module. Remember that the enveloping algebra $U$ is a quotient of the tensor algebra $T(L / \mathfrak{r})$ 3. Chapter XIII.1]. The algebra $T(L / \mathfrak{r})$ has a grading induced from the grading of $L / \mathfrak{r}$, where the degree of a product $x_{1} \otimes x_{2} \otimes \ldots \otimes x_{\nu}$ of homogeneous elements of $L / \mathfrak{r}$ is the sum of the degrees of the tensor factors $x_{1}, \ldots, x_{\nu}$. Since the relations $x \otimes y-y \otimes x=[x, y]$ "preserve" the grading, it passes to the quotient algebra $U$.

Assume that $(L / \mathfrak{r})_{k}$ is freely generated as a $\mathbb{Z}$-module by the finitely many generators $x_{k, 1}, \ldots, x_{k, n_{k}}$. We have seen before that $(\operatorname{gr}(F) / \mathfrak{R})_{k}$ is the pro- $p$ completion of $(L / \mathfrak{r})_{k}$. Therefore it is a free $\mathbb{Z}_{p}$-module generated by $x_{k, 1}, \ldots, x_{k, n_{k}}$. Observe that the grading of $\operatorname{gr}(F) / \mathfrak{R}$ gives rise to a grading of the enveloping algebra $V$ exactly as in the discrete case.

In this situation, by the Poincaré-Witt theorem [3, Theorem 3.1 in Chapter XIII], $U_{k}$ is a finitely generated free $\mathbb{Z}$-module on certain products of the $x_{k, l}$, and $V_{k}$ is a free $\mathbb{Z}_{p}$-module with the same basis. In particular, $V$ degree-wise is the pro- $p$ completion of $U$. Moreover, the product $U_{k} \times U_{l} \rightarrow U_{k+l}$ extends by continuity to $V_{k} \times V_{l} \rightarrow U_{k+l}$.

2.15. Lemma. In the above situation, $(\mathfrak{R} / \overline{[\mathfrak{R}, \mathfrak{R}]})_{k}$ is for every $k \in \mathbb{N}$ the pro-p completion of $(\mathfrak{r} /[\mathfrak{r}, \mathfrak{r}])_{k}$. 
The $U$-module multiplication

$$
U_{k} \times(\mathfrak{r} /[\mathfrak{r}, \mathfrak{r}])_{l} \rightarrow(\mathfrak{r} /[\mathfrak{r}, \mathfrak{r}])_{k+l}
$$

extends for every $k$ and $l$ by continuity to the $V$-module multiplication

$$
V_{k} \times(\mathfrak{R} / \overline{[\mathfrak{R}, \mathfrak{R}]})_{l} \rightarrow(\mathfrak{R} / \overline{[\mathfrak{R}, \mathfrak{R}]})_{k+l} .
$$

Proof. We checked that $\mathfrak{R}$ is (degree-wise) the closure of $\mathfrak{r}$ in $\operatorname{gr}(F)$. Continuity of the bracket implies (similar to the reasoning above) that the (degree-wise closed) commutator ideal $\overline{[\mathfrak{R}, \mathfrak{R}]}$ is the (degree-wise) closure of $[\mathfrak{r}, \mathfrak{r}]$. Because of Lemma 2.13 $\mathfrak{R}_{k}$ really is the pro- $p$ completion of $\mathfrak{r}$ for every $k$. Similar to the proof of Lemma 2.14, completing the exact sequence

$$
0 \rightarrow[\mathfrak{r}, \mathfrak{r}]_{k} \rightarrow \mathfrak{r}_{k} \rightarrow(\mathfrak{r} /[\mathfrak{r}, \mathfrak{r}])_{k} \rightarrow 0
$$

gives the exact sequence

$$
0 \rightarrow \overline{[\mathfrak{R}, \mathfrak{R}]}_{k} \rightarrow \mathfrak{R}_{k} \rightarrow(\mathfrak{\Re} / \overline{[\mathfrak{R}, \mathfrak{R}]})_{k} \rightarrow 0,
$$

which shows that the quotient $\mathfrak{R} / \overline{[\Re, \Re]}$ is indeed the completion of $\mathfrak{r} /[\mathfrak{r}, \mathfrak{r}]$.

The last statement follows immediately from continuity of the $V$-module multiplication map

$$
V_{k} \times(\mathfrak{R} / \overline{[\mathfrak{R}, \mathfrak{R}]})_{l} \rightarrow(\mathfrak{R} / \overline{[\mathfrak{R}, \mathfrak{R}]})_{k+l} .
$$

Continuity of this map follows from the fact that the bracket in $\operatorname{gr}(F)$ which induces this map is continuous.

2.16. Theorem. Let $w_{1}, \ldots, w_{r} \in F$ and $W_{1}, \ldots, W_{r} \in L$ be given as above, with $W_{1}, \ldots, W_{r}$ generating the ideals $\mathfrak{r}$ of $L$ and $\mathfrak{R}$ of $\operatorname{gr}(F)$. Assume that $L / \mathfrak{r}$ is a free $\mathbb{Z}$-module with enveloping algebra $U$ and that $(L / \mathfrak{r})_{k}$ is finitely generated as a $\mathbb{Z}$-module for every $k$. Assume further that $\mathfrak{r} /[\mathfrak{r}, \mathfrak{r}]$ is a free $U$-module with a basis the images of $W_{1}, \ldots, W_{r}$. in $\mathfrak{r} /[\mathfrak{r}, \mathfrak{r}]$.

Let $R$ be the closed normal subgroup of $F$ generated by $w_{1}, \ldots, w_{r}$ and set $G=$ $F / R$. Then $R / \overline{[R, R]}$ is a free $\mathbb{Z}_{p}[[G]]$-module with basis $w_{1}, \ldots, w_{r}$.

Proof. By Lemma 2.14 the assumptions imply that $\operatorname{gr}(F) / \mathfrak{R}$ is a free $\mathbb{Z}_{p}$-module. Moreover, if $V$ denotes the enveloping algebra of $\operatorname{gr}(F) / \mathfrak{R}$, then $V$ is degree-wise the pro- $p$ completion of $U$. By Lemma $[2.15 \mathfrak{R} / \overline{[\mathfrak{R}, \mathfrak{R}]}$ is degree-wise the completion of $\mathfrak{r} /[\mathfrak{r}, \mathfrak{r}]$, which is by assumption a free $U$-module freely generated by the images of $W_{1}, \ldots, W_{r}$. Since the completion of $U$ is $V$, this implies that $\mathfrak{R} / \overline{[\mathfrak{R}, \mathfrak{R}]}$ is a free $V$-module with basis the images of $W_{1}, \ldots, W_{r}$.

It follows that all the conditions of the pro- $p$ version of 11, Theorem 1 and Theorem 2] are fulfilled. As remarked at the bottom of page 52 of [11, the pro- $p$ version is obtained from the discrete version by replacing everywhere the ring of integers with $\mathbb{Z}_{p}$, the group ring $\mathbb{Z}[G]$ with the completed group algebra $\mathbb{Z}_{p}[[G]]$ and ideals and subgroups with closed ideals and closed subgroups. With these changes, the proofs of Theorem 1 and Theorem 2 of [11] almost literally remain the same to give the pro- $p$ versions of these theorems (the same is true for the pro- $p$ versions of statements and proofs in [10 which are used in [11]). In particular, $V=\operatorname{gr}\left(\mathbb{Z}_{p}[[G]]\right)$, where the filtration of $\mathbb{Z}_{p}[[G]]$ is induced by the augmentation ideal. Moreover, in the course of the proof of [11, Theorem 1] it is established that the assumptions imply that $\mathfrak{R} / \overline{[\mathfrak{R}, \mathfrak{R}]}$ is isomorphic as a $V$-module to $\operatorname{gr}(R / \overline{[R, R]})$. The latter is therefore a free $\operatorname{gr}\left(\mathbb{Z}_{p}[[G]]\right)$-module freely generated by the images $W_{1}, \ldots, W_{r}$ of $w_{1}, \ldots, w_{r}$. More details of the extension of the proof of [11, Theorem 1] are given 
in [1, after Theorem 6.14]. By Lemma 2.11$] / \overline{[R, R]}$ is a free $\mathbb{Z}_{p}[[G]]$-module with basis $w_{1}, \ldots, w_{r}$.

We are now in the situation to prove Theorem 1.4. Indeed, Theorem 1.4 follows from Proposition 2.8 if we show that $R / \overline{[R, R]}$ is a free $\mathbb{Z}_{p}[[\hat{G}]]$-module freely generated by $d-1$ elements. Remember from the proof of Proposition 2.8 that $R$ is the closed subgroup of $F:=\hat{\Gamma}_{d}$ ( $\Gamma_{d}$ is a free group on $d$ generators and $\hat{\Gamma}_{d}$ is its pro- $p$ completion) that is generated by elements $w_{1}, \ldots, w_{d-1}$ of $\hat{F}$ which are congruent to an element of $F$ modulo $\gamma_{n}(F)$ for every $n$. In particular, it follows that the images $W_{1}, \ldots, W_{d-1}$ of $w_{1}, \ldots, w_{d-1}$ in $\operatorname{gr}(F)$ belong to the subset $\operatorname{gr}\left(\Gamma_{d}\right)=L$. Moreover, $\hat{G}=F / R$.

Let $\mathfrak{r}$ be the ideal of $L$ generated by $W_{1}, \ldots, W_{d-1}$ and let $U$ be the enveloping algebra of $L / \mathfrak{r}$. By [6, Theorem 2], $L / \mathfrak{r}$ is a free $\mathbb{Z}$-module which, by [ 6 , Theorem 1], is finitely generated in each degree, and $\mathfrak{r} /[\mathfrak{r}, \mathfrak{r}]$ is a free $U$-module freely generated by the images of $W_{1}, \ldots, W_{d-1}$. Then Theorem 2.16 implies that $R / \overline{[R, R]}$ is indeed a free $\mathbb{Z}_{p}[[\hat{G}]]$-module, and Theorem 1.4 follows.

\section{REFERENCES}

1. Inga Blomer, Towards the Atiyah conjecture for link groups and their extensions, Ph.D. thesis, Georg-August-Universität Göttingen, 2007, available electronically at http://www.unimath.gwdg.de/schick/publ/Towards the Atiyah conjecture for link groups and their extensions.pdf.

2. Armand Brumer, Pseudocompact algebras, profinite groups and class formations, J. Algebra 4 (1966), 442-470. MR0202790 (34:2650)

3. Henri Cartan and Samuel Eilenberg, Homological algebra, Princeton University Press, Princeton, N. J., 1956. MR0077480 (17,1040e)

4. Adrien Douady, Cohomologie des groupes compacts totalement discontinus (d'après Tate), Séminaire Bourbaki, Vol. 5, Soc. Math. France, Paris, 1995, pp. Exp. No. 189, 287-298. MR 1603474

5. K. W. Gruenberg, Residual properties of infinite soluble groups, Proc. London Math. Soc. (3) 7 (1957), 29-62. MR0087652 (19,386a)

6. J. P. Labute, The Lie algebra associated to the lower central series of a link group and Murasugi's conjecture, Proc. Amer. Math. Soc. 109 (1990), no. 4, 951-956. MR90k:20065

7. John Labute, Mild pro-p-groups and Galois groups of p-extensions of $\mathbb{Q}$, J. Reine Angew. Math. 596 (2006), 155-182. MR2254811 (2007j:11158)

8. John P. Labute, Demuškin groups of rank $\aleph_{0}$, Bull. Soc. Math. France 94 (1966), 211-244. MR.0222177 (36:5229)

9. 142-158. MR0218495 (36:1581)

10. - On the descending central series of groups with a single defining relation, J. Algebra 14 (1970), 16-23. MR0251111 (40:4342)

11. The determination of the Lie algebra associated to the lower central series of a group, Trans. Amer. Math. Soc. 288 (1985), no. 1, 51-57. MR773046 (86b:20049)

12. Michel Lazard, Groupes analytiques p-adiques, Inst. Hautes Études Sci. Publ. Math. (1965), no. 26, 389-603. MR0209286 (35:188)

13. Peter Linnell and Thomas Schick, Finite group extensions and the Atiyah conjecture, J. Amer. Math. Soc. 20 (2007), 1003-1051. MR2328714

14. Wolfgang Lück and Holger Reich, The Baum-Connes and the Farrell-Jones conjectures in $K$ - and $L$-theory, Handbook of $K$-theory. Vols. 1, 2, Springer, Berlin, 2005, pp. 703-842. MR2181833 (2006k:19012)

15. Wilhelm Magnus, Abraham Karrass, and Donald Solitar, Combinatorial group theory: Presentations of groups in terms of generators and relations, Interscience Publishers [John Wiley \& Sons, Inc.], New York-London-Sydney, 1966. MR0207802 (34:7617) 
16. Guido Mislin and Alain Valette, Proper group actions and the Baum-Connes conjecture, Advanced Courses in Mathematics. CRM Barcelona, Birkhäuser Verlag, Basel, 2003. MR 2027168 (2005d:19007)

17. Thomas Schick, Finite group extensions and the Baum-Connes conjecture, Geometry and Topology 11 (2007), 1767-1775. MR2350467

18. Jean-Pierre Serre, Lie algebras and Lie groups, Lectures given at Harvard University, vol. 1964, W. A. Benjamin, Inc., New York-Amsterdam, 1965. MR0218496 (36:1582)

19. _ Galois cohomology, Springer-Verlag, Berlin, 1997. Translated from the French by Patrick Ion and revised by the author. MR 1466966 (98g:12007)

Mathematisches Institut, Georg-August-Universität Göttingen, Bunsenstr. 3-5, 37073 Göttingen, Germany

E-mail address: ingablomer@gmx.de

Department of Mathematics, Virginia Tech, Blacksburg, Virginia 24061-0123

E-mail address: linnell@math.vt.edu

URL: http://www.math.vt.edu/people/plinnell/

Mathematisches Institut, Georg-August-Universität Göttingen, Bunsenstr. 3-5, 37073 Göttingen, Germany

E-mail address: schick@uni-math.gwdg.de

URL: http://www. uni-math.gwdg.de/schick/ 